\title{
Editorial
}

\section{Writing and its intersections}

This issue of TEXT has by sheer happenstance become virtually a special issue on phenomenology in writing. Most of the authors of the essays in this issue direct their readers to think about the intersection of writing with the sensory domain - seeing, feeling, smelling, tasting; the whole phenomenological package. Perhaps surprisingly, given that TEXT is a journal of writing, at least half of the essay in this issue contain visual images - photographs, drawings or reproductions of famous artworks; the others rely purely on words, but for the most part explicitly reference the relationship between the body and writing/reading.

It is timely to attend to the embodied nature of writing and of reading, and to the relationship evident between images and words. The latter is a topic that emerges in the literature time and again, though it never seems to be fully resolved, while the former slips in and out of currency. On the one hand, new research, especially into cognition, reveals the centrality of our embodied status to how we think, move and find our being in the world. On the other hand, in the light of what we witness in popular culture, popular science and popular warfare it seems almost that the embodied human is going out of fashion, in favour of the possibilities offered by the digital world that inflect our understandings of what it means to be human. But as the essays in this issue suggest, we can be both, simultaneously - living, breathing, feeling and fully material subjects; and abstract 'paper' subjects who are constituted, and constitute ourselves, in language. Pierre Bourdieu sums up the complexities of our multiple being, describing:

this body which indisputably functions as the principle of individuation (in as much as it localizes in space and time, separates, isolates, etc.), ratified and reinforced by the legal definition of the individual as an abstract, interchangeable being, without qualities, is also - as a real agent, that is to say, as a habitus, with its history, its incorporated properties - ... open to the world, and therefore exposed to the world, and so capable of being conditioned by the world, shaped by the material and cultural conditions of existence in which it is placed from the beginning. (Bourdieu 2000: 133-34)

An important question related to this individuated, incorporated, legally designated and historicized subject is how such a being can know: and for many contemporary theorists, questions and problems of truth, authenticity, proof and value are negotiated through visual culture (see, e.g., Jonathan Crary in Techniques of the observer, 1990). Central to this is the recognition that visual perception is phenomenological perception: that the physical and experiential are embedded in any reading of visual material. Our elaboration of the whole world of intellectual history depends on a visual metaphor, with the word 'theory' itself coming from the Greek word teorin (to see). We 'know' what we see; but we 'know' it first because of a phenomenological response: 
seeing / knowing are concerned as much with feelings as with sentences and stories, and involves our whole being, not just our intellectual identity (Merleau-Ponty 1962: 407).

The contributors to this issue of TEXT have independently and individually come up with their own analyses of phenomenological issues in writing; with the embodied nature of the process of writing; and with ways of exploiting visual texts to illuminate or elucidate an aspect of practice or theory. Nigel Krauth and Francesca Rendle-Short explore the feeling of writing - the beat and breath of words and stories that begin in the body and make their way through to the screen or page. Krauth's essay incorporates visceral images of hatchets in feet, of transplanted organs and limbs, and of the charge that runs through the body, to recall readers to their sensory as well as their linguistic, semiotic, narrative or rational capacities. Rendle-Short, in her analysis of interpretation (whether 'authorised' or 'faulty'), lyrically outlines 'the articulation of the body' in the crafting of memory work, and the effect of writing a body in order to reclaim oneself. Josie Arnold and Kevin Brophy (in his conversation with Paul Magee) also reflect on aspects of embodiment in writing. Arnold brings in the phenomenological in her sensitive reading of Roland Barthes' The grain of the voice, where she posits the sous-voice, the articulation that emerges in a space both apophatic and liminal, an alternative way of being. Brophy describes his approach to the writing of poetry through the process of walking, of being entirely at home in place - like a fish in water - and of feeling the rhythms and patterns of speech in order to craft the lines that emerge beyond conscious thought, 'from the ends of [his] fingers'.

Let us shift now to those essays that use visual material to illustrate, elucidate or - as Andrew Melrose suggests in his work - to intervene in the authority of language. Christopher Bowman explicates his process of writing/research, the use of visual and phenomenological material to generate his work. He not only leans on the phenomenological and sensory properties of archival photographs, but travels to the sites of those photographs, putting himself in the picture as it were, to build his understanding of what it is he is investigating and producing as story. Jeri Kroll too makes use of visual material to explicate aspects of creative practice - in this case, by rehearsing the Icarus trope and its deployment in both visual and poetic modes. Kroll's analysis of this suggests the possibility of our leaping, like Icarus, into uncharted space in order to find out what we do not know; to interrupt the established research pathways; and to map 'realms that are yet to come'. Andrew Melrose likewise looks to visual (and to audio) texts to intervene in the established processes of storytelling and storymaking. Using examples from his own life and practice, he takes readers on a story-journey and, along the way, demonstrates the continued value of authenticity and critical engagement in writing. Writing from a perspective informed by pedagogy, Susan O'Rourke and Josephine Ellis show how the teaching of creative writing can illuminate and invigorate professional practice for their communication students: particularly when that creative writing is associated with visual texts. By exposing their students to art, and providing them techniques to respond creatively to those images, they suggest, more effective and perhaps more ethical communication skills can be transferred.

Stephanie Green's essay similarly focuses on the domain of teaching, and of finding ways to transfer knowledge about creative and ethic practices - in her case, to students in regional Australia. For Green, encounters with language, culture and place, and awareness of the particularity of experience, can usefully be mined to give students access to knowing, and to making, in ways that will allow them to find their own futures. The particularity, or materiality, of context also informs Stephen Muecke's essay, which moves through current issues of policy and procedure to produce an argument about how writers might 
position themselves, and both create and address their readership. Central to this is the question of knowledge, and of the ontology of creative texts. Are they knowledge-texts, or 'mere entertainment'? If they are outcomes from research, what knowledge do they deliver, for whom, and how do they disseminate it? And finally, Donna Lee Brien, who is in the process of writing a history of the AAWP, sets out an account of the relevant field - its complexities and perplexities, the risks of tedium or of hagiography - and, drawing on the literature of organisational studies and its history, suggests both the reason for this planned publication, and appropriate ways of approaching the task.

Knowledge, embodiment, practice, pedagogy, research ... these are questions that have been engaging the attention of creative academics at least since the early 1990s, and that have taken on increased urgency thanks to recent government initiatives. For many Australian academics, the Excellence in Research for Australia project (ERA) finds its way into their daily work lives and, for some unlucky people, into their sleep as well. Creative writing, which is categorized as part of the Humanities and Creative Arts cluster, was evaluated in a trial run of the ERA during 2009. A non-scientific, indeed largely anecdotal, investigation of the outcomes of that trial (i.e., we asked friends at other universities how their programs scored) suggests that writing, by and large, was not very highly regarded as a field of research (see Appendix). We could complain that humanities academics do not, by and large, understand our research paradigm; we could point out that the failure to collect evidence of creative outputs over the past decade has disadvantaged us; or perhaps we could turn our thoughts to creative ways of articulating and recording knowledge in, through and about creative writing. We noted at the start of this editorial the significance of visual texts in the process of knowledge-making and reading. Alongside this is the understanding that when we see and make sense of what we see, we use our whole body. It is not just linguistic ability, rational skills or conscious reason, but all our sensory capacities that are put to work in 'reading' visual material: something often overlooked by writers, embedded as we are in the world of words. Possibly greater attention to the opportunities for knowledge generation and dissemination that are offered by the sorts of interventions discussed in these essays - visual, phenomenological - might provide a way through the problem of finding a bureaucratic fit.

Jen Webb

Nigel Krauth

\section{List of works cited}

Bourdieu, Pierre 2000 Pascalian Meditations (trans R Nice), Cambridge:

Policy Press return to text

Crary, Jonathan 1990 Techniques of the Observer: On Vision and Modernity in the Nineteenth Century, Cambridge MA: MIT Press return to text Merleau-Ponty, Maurice 1962 Phenomenology of Perception (trans C Smith) London: Routledge \& Kegan Paul return to text

\section{Appendix}

The email Q\&A asked the following questions:

- Did your CW area make submissions to the 2009 ERA trial?

- Were you personally involved in coordinating the process of gathering staff Research Statements, etc? 
- Were you confident that your $\mathrm{CW}$ area had a strong claim to make regarding its $\mathrm{CW}$ output?

- Why were you confident (or not)?

- Was there clear understanding in your $\mathrm{CW}$ area regarding previous academic debate about CW as research?

- Was your $\mathrm{CW}$ area ready for the $\mathrm{ARC}$ announcement that $\mathrm{CW}$ would be treated as research, subject to review processes?

- Were you happy with the idea of the Research Statement to accompany CW products?

- Were you happy with the idea of ARC committee review of CW products?

- Following the announcement of results from the 2009 trial in December, what has been your institution's response regarding CW's performance?

- Were you happy with the ranking given to your institution in FoR 1904 in the trial?

- Are you planning already for ERA 2010? What have you learnt from the 2009 HCA trial?

- Has your institution sworn you to secrecy regarding any of these matters?

Responses came from CW academics in five states (Western Australia, South Australia, Victoria, New South Wales and Queensland). These responses covered universities that did 'very well' in the trial, did 'very poorly', or didn't make a submission at all.

There were just 8 responses, but they were from significant players in the field. Here are our interpretations of the responses to each question:

- Did your CW area make submissions to the 2009 ERA trial? Most did, but some did not, either due to not meeting the submissions number threshold, or, more worrying, because CW was allegedly not valued sufficiently in the university and therefore was 'deliberately excluded' from the trial.

- Were you personally involved in coordinating the process of gathering staff Research Statements, etc?

Most respondents were involved. But one, a Head of a creative program, was excluded from the process. In later parts of her/his response s/he indicates that the institution, upon receiving results of the trial, has changed its tactics for the 2010 round. Seemingly it has learnt that all stakeholders in the hierarchy need to be involved.

- Were you confident that your $C W$ area had a strong claim to make regarding its $C W$ output?

Only one respondent was super-confident, and her/his confidence was justified by an outstanding final result. All other respondents indicated doubts of one sort or another. Several respondents answered this question with 'Yes and No', but in their later responses there was more disappointment in the result than elation.

- Why were you confident (or not)?

Confidence appears to revolve around departmental set-up and personnel. The situation of $\mathrm{CW}$ in the school, or the institution overall, seems to play a significant part regarding confidence. Numbers of $\mathrm{CW}$ staff and their known rates of production are factors. While confidence in the ERA process reflects the confidence of the CW staff in their setting, for some, ERA was a way of drawing the university's attention to previously-ignored good work being done. 
- Was there clear understanding in your CW area regarding previous academic debate about $C W$ as research?

There were mixed responses to this question. Some areas were savvy, others not at all. It seems the concept of CW as research has been discussed for years in some departments, but rarely in others.

- Was your $C W$ area ready for the ARC announcement that $C W$ would be treated as research, subject to review processes?

There were mixed responses to this question too. One respondent summed it up by saying: 'Yes in terms of knowledge. No in terms of preparation for submissions.'

- Were you happy with the idea of the Research Statement to accompany CW products?

Generally the Research Statement was seen as a new territory, and respondents were cautious about entering it. Some saw it as an imposition upon the creative work's natural and obvious research qualities. Most saw it as inevitable in the circumstance.

- Were you happy with the idea of ARC committee review of $C W$ products? There was strong reaction regarding this item. Most questioned the reputation of the ARC to undertake the task, considering its non-support of creativity in the past. It seems that the ARC has not publicised well its methods and its concern to ensure good practice in the area of refereeing of creative submissions.

- Following the announcement of results from the 2009 trial in December, what has been your institution's response regarding $C W$ 's performance? Responses ranged from completely dismissive to supportive. In some supportive cases, the university had only expressed itself thus following CW staff pointedly making the case regarding the trial results. It seems we need to keep CW continually in our administrators' purview, for them to realise what's happening.

- Were you happy with the ranking given to your institution in FoR 1904 in the trial?

There were few happy responses.

- Are you planning already for ERA 2010? What have you learnt from the 2009 HCA trial?

All responses indicated that plans were in place to deal with the 2010 round in a more focused way. In all cases this involved a greater ownership of the process by the CW staff themselves.

- Has your institution sworn you to secrecy regarding any of these matters?

Some said they were sworn to confidentiality. They also noted that they felt this was inappropriate. return to text 


\section{TEXT}

Vol 14 No 1 April 2010

http://www.textjournal.com.au

Editors: Nigel Krauth \& Jen Webb

Text@griffith.edu.au 